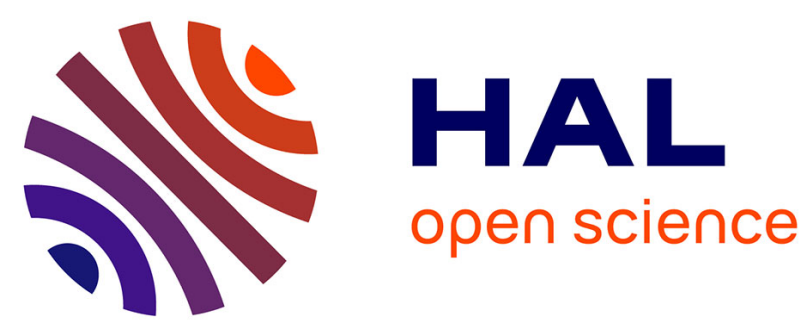

\title{
Carleman estimates for the one-dimensional heat equation with a discontinuous coefficient and applications
}

Assia Benabdallah, Yves Dermenjian, Jérôme Le Rousseau

\section{- To cite this version:}

Assia Benabdallah, Yves Dermenjian, Jérôme Le Rousseau. Carleman estimates for the onedimensional heat equation with a discontinuous coefficient and applications. Comptes Rendus Mécanique, 2006, 334 (10), pp.582-586. 10.1016/j.crme.2006.07.001 . hal-00463326

\section{HAL Id: hal-00463326 \\ https://hal.science/hal-00463326}

Submitted on 11 Mar 2010

HAL is a multi-disciplinary open access archive for the deposit and dissemination of scientific research documents, whether they are published or not. The documents may come from teaching and research institutions in France or abroad, or from public or private research centers.
L'archive ouverte pluridisciplinaire HAL, est destinée au dépôt et à la diffusion de documents scientifiques de niveau recherche, publiés ou non, émanant des établissements d'enseignement et de recherche français ou étrangers, des laboratoires publics ou privés. 


\title{
Carleman estimates for the one-dimensional heat equation with a discontinuous coefficient and applications
}

\author{
Assia Benabdallah, Yves Dermenjian, Jérôme Le Rousseau ${ }^{1}$ \\ Laboratoire d'Analyse Topologie Probabilités, CNRS UMR 6632, Université d'Aix-Marseille I \\ 39 rue F. Joliot-Curie, 13453 Marseille
}

\begin{abstract}
We study the observability and some of its consequences for the one-dimensional heat equation with a discontinuous coefficient (piecewise $\mathscr{C}^{1}$ ). The observability, for a linear equation, is obtained by a Carleman-type estimate. This kind of observability inequality yields results of controllability to the trajectories for semilinear equations. It also yields a stability result for the inverse problem of the identification of the diffusion coefficient. To cite this article: A. Benabdallah, Y. Dermenjian, J. Le Rousseau, C. R. Mécanique - (2006).
\end{abstract}

\section{Résumé}

Inégalité de Carleman pour une équation de la chaleur à coefficient discontinu et applications. On étudie l'observabilité et certaines de ses conséquences pour une équation de la chaleur à coefficient discontinu $\left(\mathscr{C}^{1}\right.$ par morceaux). L'observabilité, pour une équation linéaire, est obtenue au moyen d'inégalités de Carleman. Ce type d'inégalités d'observabilité permet d'obtenir des résultats de contrôlabilité aux trajectoires pour des équations semi-linéaires. Elle permet aussi de résoudre un problème inverse d'identification et de stabilité pour le coefficient de diffusion. Pour citer cet article : A. Benabdallah, Y. Dermenjian, J. Le Rousseau, C. R. Mécanique - (2006).

Key words: Carleman estimate; non-smooth coefficients ; parabolic equations ; control ; inverse problem.

Mots-clés : Inégalité de Carleman; coefficients non réguliers; équations paraboliques ; contrôle; problème inverse.

\section{Introduction}

The question of controllability of partial differential systems with discontinuous coefficients and its dual counterpart, observability, are not fully solved yet. Recently, a result of controllability for a semilinear

\footnotetext{
Email addresses: assia@cmi.univ-mrs.fr (Assia Benabdallah), dermenji@cmi.univ-mrs.fr (Yves Dermenjian), jlerous@cmi.univ-mrs.fr (Jérôme Le Rousseau).

1 Corresponding author.
} 
heat equation with a discontinuous coefficient was proven in [1] by means of a Carleman observability estimate. Roughly speaking, as in the case of hyperbolic systems (see e.g. [2, page 357]), the authors of [1] proved their controllability result in the case where the control is supported in the region where the diffusion coefficient is the 'lowest'. In both cases, however, the approximate controllability holds without any restriction on the 'monotonicity' of the coefficients. It is then natural to question whether or not an observability estimate holds in the case of non-smooth coefficients and arbitrary observation location.

In the one-dimensional case, the controllability result for linear parabolic equations was proven for coefficients with bounded variations in [3]. The proof relies on Russel's method [4]. However, the question of the existence of a Carleman-type observability estimate remains open. The present paper provides a positive answer in the case of piecewise $\mathscr{C}^{1}$ coefficients.

Carleman estimates for parabolic equations with smooth coefficients were proven in [5]. The proof is based on the construction of suitable weight functions $\beta$ whose gradient is non-zero in the complement of the observation region. In the non-smooth case, in [1], to obtain the observability, the authors have to add the assumptions on the 'monotonicity' of the coefficients mentioned above. In both cases, the weight function $\beta$ was chosen in the domain of the operator $\nabla \cdot(c \nabla)$. Here, we do not impose this constraint, which, with the jump of the derivative of $\beta$ as a new parameter, enables us to control the interface terms in the derivation of the Carleman estimate and therefore allows us to relax the 'monotonicity' condition on the coefficient. Note however that the results of [1] are for the multidimensional heat equation. The relaxation of the 'monotonicity' condition in the $n$-dimensional case, $n \geq 2$, remains, to our knowledge, open.

With such a Carleman estimate at hand, we treat the problem of the null controllability for classes of semilinear parabolic equations of the form

$$
\begin{cases}\partial_{t} y-\partial_{x}\left(c \partial_{x} y\right)+\mathscr{G}(y)=1_{\omega} v & \text { in } Q, \\ y(t, x)=0 & \text { on } \Sigma, \\ y(0, x)=y_{0}(x) & \text { in } \Omega,\end{cases}
$$

where $T>0, \Omega=(0,1), Q=(0, T) \times \Omega, \Sigma=(0, T) \times\{0,1\}$ and where $\mathscr{G}: \mathbb{R} \rightarrow \mathbb{R}$ is locally Lipschitz and $\mathscr{G}(0)=0$ (further assumptions on the nonlinear function $\mathscr{G}$ or on the initial condition will be introduced below).

We also provide a stability result for the inverse problem of the identification of the diffusion coefficient.

\section{Global Carleman estimates}

We consider a piecewise $\mathscr{C}^{1}$ diffusion coefficient with a finite number of singularities. We shall thus

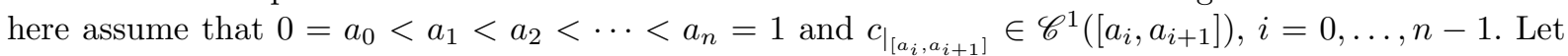
$j \in\{0, \ldots, n-1\}$ be fixed in the sequel and $\omega_{0} \Subset \omega \Subset\left(a_{j}, a_{j+1}\right)$ be a non-empty open set. Let $T>0$. We shall use the following notations $S=\left\{a_{1}, \ldots, a_{n-1}\right\}, \Omega^{\prime}=\Omega \backslash\left\{a_{1}, \ldots, a_{n-1}\right\}, Q=(0, T) \times \Omega$, and $Q^{\prime}=(0, T) \times \Omega^{\prime}$.

We first introduce a particular type of weight functions, which are constructed using the following lemma.

Lemma 2.1 There exists a function $\widetilde{\beta} \in \mathscr{C}(\Omega)$ such that $\widetilde{\beta}_{\left.\right|_{\left[a_{i}, a_{i+1}\right]}} \in \mathscr{C}^{2}\left(\left[a_{i}, a_{i+1}\right]\right), i=0, \ldots, n-1$, satisfying $\widetilde{\beta}>0$ in $\Omega, \widetilde{\beta}=0$ on $\{0,1\},\left(\widetilde{\beta}_{\left.\right|_{\left[a_{j}, a_{j+1}\right]}}\right)^{\prime} \neq 0$ in $\left[a_{j}, a_{j+1}\right] \backslash \omega_{0},\left(\widetilde{\beta}_{\left.\right|_{\left[a_{i}, a_{i+1}\right]}}\right)^{\prime} \neq 0, i \in\{0, \ldots, n-1\}$, $i \neq j$, and the function $\widetilde{\beta}$ satisfies the following trace properties: for some $\alpha>0,\left(A_{i} u, u\right) \geq \alpha|u|^{2}, u \in \mathbb{R}^{2}$, with the matrices $A_{i}$, defined by 


$$
A_{i}=\left(\begin{array}{ll}
{\left[\widetilde{\beta}^{\prime}\right]_{a_{i}}} & \widetilde{\beta}^{\prime}\left(a_{i}^{+}\right)\left[c \widetilde{\beta}^{\prime}\right]_{a_{i}} \\
\widetilde{\beta^{\prime}}\left(a_{i}^{+}\right)\left[c \widetilde{\beta}^{\prime}\right]_{a_{i}} & \widetilde{\beta}^{\prime}\left(a_{i}^{+}\right)\left[c \widetilde{\beta}^{\prime}\right]_{a_{i}}^{2}+\left[c^{2}\left(\widetilde{\beta}^{\prime}\right)^{3}\right]_{a_{i}}
\end{array}\right), \quad i=1, \ldots, n-1 .
$$

where $[\rho]_{x}=\rho\left(x^{+}\right)-\rho\left(x^{-}\right)$for $x \in(0,1)$.

Choosing a function $\widetilde{\beta}$, as in the previous lemma, we introduce $\beta=\widetilde{\beta}+K$ with $K=m\|\widetilde{\beta}\|_{\infty}$ and $m>1$. For $\lambda>0$ and $t \in(0, T)$, we define the following weight functions

$$
\varphi(t, x)=\frac{e^{\lambda \beta(x)}}{t(T-t)}, \quad \eta(t, x)=\frac{e^{\lambda \bar{\beta}}-e^{\lambda \beta(x)}}{t(T-t)},
$$

with $\bar{\beta}=2 m\|\widetilde{\beta}\|_{\infty}$ (see [1]). We introduce

$$
\begin{aligned}
\aleph=\left\{q \in \mathscr{C}(Q, \mathbb{R}) ; q_{[0, T] \times\left[a_{i}, a_{i+1}\right]}\right. & \in \mathscr{C}^{2}\left([0, T] \times\left[a_{i}, a_{i+1}\right]\right), i=0, \ldots, n-1, \\
q_{\left.\right|_{\Sigma}}=0, & \text { and } q \text { satisfies (TC), for all } t \in(0, T)\},
\end{aligned}
$$

with

$$
q\left(a_{i}^{-}\right)=q\left(a_{i}^{+}\right), \quad c\left(a_{i}^{-}\right) \partial_{x} q\left(a_{i}^{-}\right)=c\left(a_{i}^{+}\right) \partial_{x} q\left(a_{i}^{+}\right), i=1, \ldots, n-1 .
$$

Theorem 2.2 There exist $\lambda_{1}=\lambda_{1}(\Omega, \omega)>0, s_{1}=\left(T+T^{2}\right) \widetilde{s}_{1}>0$ and a positive constant $C=C(\Omega, \omega)$ so that the following estimate holds

$$
\begin{gathered}
s^{-1} \iint_{Q} e^{-2 s \eta} \varphi^{-1}\left(\left|\partial_{t} q\right|^{2}+\left|\partial_{x}\left(c \partial_{x} q\right)\right|^{2}\right) d x d t+s \lambda^{2} \iint_{Q} e^{-2 s \eta} \varphi\left|\partial_{x} q\right|^{2} d x d t+s^{3} \lambda^{4} \iint_{Q} e^{-2 s \eta} \varphi^{3}|q|^{2} d x d t \\
+s \lambda \sum_{i=1}^{n-1} \int_{0}^{T} \varphi\left(t, a_{i}\right) e^{-2 s \eta\left(t, a_{i}\right)}\left|\partial_{x} q\left(t, a_{i}^{-}\right)\right|^{2} d t+s^{3} \lambda^{3} \sum_{i=1}^{n-1} \int_{0}^{T} \varphi^{3}\left(t, a_{i}\right) e^{-2 s \eta\left(t, a_{i}\right)}\left|q\left(t, a_{i}\right)\right|^{2} d t \\
\leq C\left[s^{3} \lambda^{4} \iint_{(0, T) \times \omega} e^{-2 s \eta} \varphi^{3}|q|^{2} d x d t+\iint_{Q} e^{-2 s \eta}\left|\partial_{t} q \pm \partial_{x}\left(c \partial_{x} q\right)\right|^{2} d x d t\right], \quad(3)
\end{gathered}
$$

for $s \geq s_{1}, \lambda \geq \lambda_{1}$ and for all $q \in \aleph$.

Proof. Arguing as in [5,1], we set $\psi=e^{-s \eta} q$. We obtain, in the derivation of the Carleman estimate, integral terms over $Q^{\prime}$ and some time integrals over $(0, T)$ with trace terms at $a_{i}, i=1, \ldots, n-1$. In fact, the leading order terms for these time integrals at $a_{i}$ (w.r.t. to the parameters $s$ and $\lambda$ ) are given by

$$
\mu_{i}:=s \lambda \int_{0}^{T} \varphi\left(t, a_{i}\right)\left[\beta^{\prime}\left|c \partial_{x} \psi\right|^{2}(t, .)\right]_{a_{i}} d t+s^{3} \lambda^{3}\left[c^{2}\left(\beta^{\prime}\right)^{3}\right]_{a_{i}} \int_{0}^{T} \varphi^{3}\left(t, a_{i}\right)\left|\psi\left(t, a_{i}\right)\right|^{2} d t, \quad i=1, \ldots, n-1 .
$$

We obtain $\mu_{i}=s \lambda \int_{0}^{T} \varphi\left(t, a_{i}\right)\left(A_{i} u\left(t, a_{i}\right), u\left(t, a_{i}\right)\right) d t$ with $u\left(t, a_{i}\right)=\left(c\left(a_{i}^{-}\right) \partial_{x} \psi\left(t, a_{i}^{-}\right), s \lambda \varphi\left(t, a_{i}\right) \psi\left(t, a_{i}\right)\right)^{t}$, with the $2 \times 2$ matrix $A_{i}$ as in Lemma 2.1. This term is thus positive and can 'absorb' the remaining time integrals at $a_{i}$ if we choose the parameters $s$ and $\lambda$ to be sufficiently large. The rest of the proof can be adapted from $[5,1]$.

Remark 1 In a similar fashion, we can obtain Carleman estimates with a 'side observation' for the operators $\partial_{t} \pm \partial_{x}\left(c \partial_{x}\right)$, for instance with the term

$$
s \lambda \int_{0}^{T} \varphi(t, 0) e^{-2 s \eta(t, 0)}\left|\partial_{x} q\right|^{2}(t, 0) d t
$$

replacing the volume integral over $(0, T) \times \omega$ on the r.h.s. of (3). To this purpose, we need to introduce a weight function $\widetilde{\beta}$ according to the following lemma. 
Lemma 2.3 There exists a function $\widetilde{\beta} \in \mathscr{C}(\Omega)$ such that $\widetilde{\beta}_{\left.\right|_{\left[a_{i}, a_{i+1}\right]}} \in \mathscr{C}^{2}\left(\left[a_{i}, a_{i+1}\right]\right), i=0, \ldots, n-1$, satisfying $\widetilde{\beta}>0$ in $\Omega, \widetilde{\beta}(1)=0,\left(\widetilde{\beta}_{\left.\right|_{\left[a_{i}, a_{i+1}\right]}}\right)^{\prime} \leq \nu<0, i \in\{0, \ldots, n-1\}$, and the function $\widetilde{\beta}$ satisfies the following trace properties: for some $\alpha>0,\left(A_{i} u, u\right) \geq \alpha|u|^{2}, u \in \mathbb{R}^{2}$ with the matrices $A_{i}$, defined as in Lemma 2.1.

Remark 2 Note that an inequality, of the form of (3), with these pointwise terms on the l.h.s of the Carleman estimates can still be obtained in the case of a smooth coefficient by simply choosing the weight function $\beta$ to have a jump condition for its derivative and satisfying the properties given by Lemma 2.1.

\section{Controllability results}

The Carleman estimate (3) allows to give observability estimates that yield results of controllability to the trajectories for classes of semilinear heat equations. Considering a piecewise $\mathscr{C}^{1}$ diffusion coefficient with $n-1$ points of discontinuities, $a_{1}, \ldots, a_{n-1}$, with $0=a_{0}<a_{1}<\cdots<a_{n-1}<1=a_{n}$. We let $\omega \Subset\left(a_{j}, a_{j+1}\right)$ be an non-empty open set for some $j \in\{0, \ldots, n-1\}$. We first state an observability result with an $L^{2}$ observation. We let $a$ be in $L^{\infty}(Q)$ and $q_{T} \in L^{2}(\Omega)$. From Carleman estimate (3) we obtain the following proposition.

Proposition 3.1 The solution $q$ to

$$
\begin{cases}-\partial_{t} q-\partial_{x}\left(c \partial_{x} q\right)+a q=0 & \text { in } Q \\ q=0 & \text { on } \Sigma, \\ q(T)=q_{T} & \text { in } \Omega\end{cases}
$$

satisfies $\|q(0)\|_{L^{2}(\Omega)}^{2} \leq e^{C K\left(T,\|a\|_{\infty}\right)} \iint_{(0, T) \times \omega}|q|^{2} d x d t$, where $K\left(T,\|a\|_{\infty}\right)=1+\frac{1}{T}+T\|a\|_{\infty}+\|a\|_{\infty}^{2 / 3}$.

The proof of this proposition can be found in [1]. Such an observability estimate yields the null controllability of the following semilinear parabolic equation (1) where $\mathscr{G}: \mathbb{R} \rightarrow \mathbb{R}$ is locally Lipschitz and $\mathscr{G}(0)=0$. This implies that $\mathscr{G}(s)=s g(s)$, with $g$ in $L_{\text {loc }}^{\infty}(\mathbb{R})$.

Theorem 3.1 Let $c$ be a piecewise $\mathscr{C}^{1}$ diffusion coefficient with $n-1$ points of discontinuities, $0<a_{1}<$ $\cdots<a_{n-1}<1$. We let $\omega \Subset\left(a_{j}, a_{j+1}\right)$ be an non-empty open set and we assume that $\mathscr{G}$ is locally Lipschitz. Let $T>0$ :

(i) Local null controllability: There exists $\varepsilon>0$ such that for all $y_{0}$ in $L^{2}(\Omega)$ with $\left\|y_{0}\right\|_{L^{2}(\Omega)} \leq \varepsilon$, there exists a control $v \in L^{2}((0, T) \times \omega)$ such that the corresponding solution to system (1) satisfies $y(T)=0$.

(ii) Global null controllability: Let $\mathscr{G}$ satisfy in addition $\lim _{|s| \rightarrow \infty} \frac{|\mathscr{G}(s)|}{|s| \ln ^{3 / 2}(1+|s|)}=0$. Then for all $y_{0}$ in $L^{2}(\Omega)$, there exists $v \in L^{2}((0, T) \times \omega)$ such that the solution to system (1) satisfies $y(T)=0$.

The proof is based on a fixed point argument and is along the same lines as that in [6] and originates from [7].

Remark 3 A similar result holds for a boundary control, i.e., by imposing $y=v$ at either 0 or 1 , with $v \in L^{2}(0, T)$. Note that as usual, $y(T)=y^{*}(T)$ can replace $y(T)=0$ in the previous statements, where $y^{*}$ is any trajectory defined in $[0, T]$ of system (1), corresponding to some initial data $y_{0}^{*} \in L^{2}(\Omega)$ and any $v^{*}$ in $L^{2}((0, T) \times \omega)\left(L^{2}(0, T)\right.$ in the case of a boundary control). For the local controllability result, one has to assume $\left\|y_{0}-y_{0}^{*}\right\|_{L^{2}(\Omega)} \leq \varepsilon$, with $\varepsilon$ sufficiently small. 


\section{Stability for a discontinuous diffusion coefficient}

In [8], the authors establish a uniqueness result for the discontinuous diffusion coefficient $c$ as well as a stability inequality. This inequality estimates the discrepancy in the coefficients $c$ and $\widetilde{c}$ of two materials (with the same geometry) with an upper bound given by some Sobolev norms of the difference between the solutions $y$ and $\widetilde{y}$ to

$$
\left\{\begin{array} { l l } 
{ \partial _ { t } \widetilde { y } - \partial _ { x } ( \widetilde { c } \partial _ { x } \widetilde { y } ) = 0 } & { \text { in } Q , } \\
{ \widetilde { y } ( t , x ) = h ( t , x ) } & { \text { on } \Sigma , } \\
{ \widetilde { y } ( 0 , x ) = \widetilde { y } _ { 0 } ( x ) } & { \text { in } \Omega , }
\end{array} \quad \left\{\begin{array}{ll}
\partial_{t} y-\partial_{x}\left(c \partial_{x} y\right)=0 & \text { in } Q, \\
y(t, x)=h(t, x) & \text { on } \Sigma, \\
y(0, x)=y_{0}(x) & \text { in } \Omega .
\end{array}\right.\right.
$$

Set $u=y-\widetilde{y}$ and $q=\partial_{t} u$. Then $q$ is solution to the following problem

$$
\begin{cases}\partial_{t} q-\partial_{x}\left(c \partial_{x} q\right)=\partial_{x}\left((c-\widetilde{c}) \partial_{x} \partial_{t} \widetilde{y}\right) & \text { in } Q^{\prime}, \\ q=0 & \text { on } \Sigma, \\ \text { transmission conditions }\left(\mathrm{TC}_{g}\right) & \text { on } S \times[0, T],\end{cases}
$$

with

$$
q\left(x^{-}\right)=q\left(x^{+}\right), \quad\left(c \partial_{x} q\right)\left(x^{-}\right)=\left(c \partial_{x} q\right)\left(x^{+}\right)+g(x, t),
$$

where $x \in S=\left\{a_{1}, \ldots, a_{n-1}\right\}$, the set of singularities for both $c$ and $\widetilde{c}$, and

$$
g(x, t)=\left((c-\widetilde{c}) \partial_{x} \partial_{t} \widetilde{y}\right)\left(x^{+}\right)-\left((c-\widetilde{c}) \partial_{x} \partial_{t} \widetilde{y}\right)\left(x^{-}\right) .
$$

If the solutions $y$ and $\widetilde{y}$ to (5) satisfy some (regularity) conditions (that can be achieved with some choices of boundary conditions $h$ and initial conditions $y_{0}$ and $\widetilde{y}_{0}$ in $L^{2}(\Omega)$ - see [8] for details) we have the following stability result.

Theorem 4.1 We assume that the diffusion coefficients $c$ and $\widetilde{c}$ are piecewise constant with the same singularity locations. Then there exists a constant $C$ such that

$$
|c-\widetilde{c}|_{L^{\infty}(\Omega)}^{2} \leq C\left|\partial_{x}\left(\partial_{t} y-\partial_{t} \widetilde{y}\right)(., 0)\right|_{L^{2}(0, T)}^{2}+C\left|\Delta y\left(T^{\prime}, .\right)-\Delta \widetilde{y}\left(T^{\prime}, .\right)\right|_{L^{2}\left(\Omega^{\prime}\right)}^{2},
$$

where $\Omega^{\prime}$ is the open set $\Omega$ with the singularities of c removed.

A Carleman estimate is the key ingredient in the proof of such a stability estimate. In [8], this Carleman estimate was proven in any dimension but with an additional 'monotonicity' assumption on the discontinuous diffusion coefficient. In the present case, we can establish such a Carleman estimate for a piecewise $\mathscr{C}^{1}$ diffusion coefficient. Choosing the weight function as in Lemma 2.3, we have the following estimate.

Theorem 4.2 Let $t_{0}>0$, in $(0, T)$ and $g\left(., a_{i}\right) \in H^{1}\left(t_{0}, T\right), i=1, \ldots, n-1$. There exist $\lambda_{1}>1$, $s_{1}=s_{1}\left(\lambda_{1}\right)>0$ and a positive constant $C$ so that the following estimate holds

$$
\begin{array}{r}
\left|M_{1}\left(e^{-s \eta} q\right)\right|_{L^{2}\left(Q^{\prime}\right)}^{2}+\left|M_{2}\left(e^{-s \eta} q\right)\right|_{L^{2}\left(Q^{\prime}\right)}^{2}+s \lambda^{2} \iint_{Q} e^{-2 s \eta} \varphi\left|\partial_{x} q\right|^{2} d x d t+s^{3} \lambda^{4} \iint_{Q} e^{-2 s \eta} \varphi^{3}|q|^{2} d x d t \\
\leq C\left[s \lambda \int_{t_{0}}^{T} e^{-2 s \eta} \varphi\left|\partial_{x} q\right|^{2}(t, 0) d t+\iint_{Q} e^{-2 s \eta}\left|\partial_{t} q \pm \partial_{x}\left(c \partial_{x} q\right)\right|^{2} d x d t+s \lambda \int_{t_{0}}^{T} \int_{S} e^{-2 s \eta} \varphi|g|^{2} d \sigma d t\right. \\
\left.\quad+\int_{t_{0}}^{T} \int_{S} e^{-2 s \eta} \varphi^{4}|g|^{2} d \sigma d t+s^{-2} \int_{t_{0}}^{T} \int_{S} e^{-2 s \eta}\left|\partial_{t} g\right|^{2} d \sigma d t\right],
\end{array}
$$

for $s \geq s_{1}, \lambda \geq \lambda_{1}$ and for all $q \in \aleph_{g}$, with $M_{1}$ and $M_{2}$ given by

$$
M_{1} \psi=\partial_{x}\left(c \partial_{x} \psi\right)+s^{2} \lambda^{2} \varphi^{2}\left(\beta^{\prime}\right)^{2} c \psi+s\left(\partial_{t} \eta\right) \psi, \quad M_{2} \psi=\partial_{t} \psi-2 s \lambda \varphi c \beta^{\prime} \partial_{x} \psi-2 s \lambda^{2} \varphi c\left(\beta^{\prime}\right)^{2} \psi,
$$


and $\aleph_{g}$ is given by

$$
\begin{aligned}
\aleph_{g}=\left\{q \in H^{1}\left(t_{0}, T, H_{0}^{1}(\Omega)\right) ; q_{\left.\right|_{\left(t_{0}, T\right) \times\left(a_{i}, a_{i+1}\right)}} \in L^{2}\left(t_{0}, T, H^{2}\left(\left(a_{i}, a_{i+1}\right)\right)\right.\right. \\
\left.\quad i=0, \ldots, n-1, \quad q_{\left.\right|_{\Sigma}}=0 \text { and } q \text { satisfies }\left(T C_{g}\right) \text { a.e. w.r.t. } t\right\} .
\end{aligned}
$$

Remark 4 Observe that in Theorem 4.1 and Theorem 4.2, we need not assume that jumps for $c$ are greater than some positive constants $\Delta$ at its points of discontinuities, as is done in [8]. This is due to the choice made on the weight function $\widetilde{\beta}$ in Lemma 2.3. This remark is to be connected to the proof of Theorem 1.3 in [8, estimate (1.16) and following arguments].

\section{References}

[1] A. Doubova, A. Osses, J.-P. Puel, Exact controllability to trajectories for semilinear heat equations with discontinuous diffusion coefficients, ESAIM: COCV 8 (2002) 621-661.

[2] J.-L. Lions, Contrôlabilité exacte Perturbations et Stabilisation de systèmes distribués, Vol. 1, Masson, Paris, 1988.

[3] E. Fernández-Cara, E. Zuazua, On the null controllability of the one-dimensional heat equation with $B V$ coefficients, Comput. Appl. Math. 21 (2002) 167-190.

[4] D L. Russel, A unified boundary controllability theory for hyperbolic and parabolic partial differential equations, Studies in Appl. Math., 52 (1973) 189-221.

[5] A. Fursikov, O. Yu. Imanuvilov, Controllability of evolution equations, Seoul National University, Korea, Lecture notes 34 (1996).

[6] A. Doubova, E. Fernandez-Cara, M. Gonzales-Burgos, E. Zuazua, On the controllability of parabolic systems with a nonlinear term involving the state and the gradient, SIAM J. Control Optim. 41 (2002) 798-819.

[7] E. Fernández-Cara, E. Zuazua, Null and approximate controllability for weakly blowing up semilinear heat equations, Ann. Inst. H. Poincaré, Analyse non lin. 17 (2000) 583-616.

[8] A. Benabdallah, P. Gaitan, J. Le Rousseau, An inverse problem for the heat equation with discontinuous diffusion coefficients, Preprint: LATP, Universités de Marseille, www.cmi.univ-mrs.fr/ jlerous/publications.html (2005). 